\title{
System Level Evaluation of LTE Networks with Semidistributed Intercell Interference Coordination
}

\author{
D. González, S. Ruiz, M. García-Lozano, J. Olmos, A. Serra \\ Dept. of Signal Theory and Communications \\ Universitat Politècnica de Catalunya (UPC). Barcelona, Spain \\ e-mail: \{david.gonzalez.gonzalez, silvia.ruiz\}@upc.edu
}

\begin{abstract}
GPP LTE is the evolution of UMTS which will make possible to deliver high quality multimedia services with an improved user experience. Since Radio Resource Management (RRM) has been recognized as a key point to successfully accomplish this target, the performance evaluation of a multi-cell resource allocation scheme applied to LTE downlink (DL) is presented in this paper. A semi-distributed RRM framework is discussed and evaluated from a system level viewpoint. Detailed link level simulations have also been carried out to properly back up the results.
\end{abstract}

Keywords- ICIC, LTE system level evaluation, cell engineering.

\section{INTRODUCTION}

3GPP LTE is the evolution of the UMTS which will make possible to deliver next generation high quality multimedia services according to the users' expectations [1]. OFDM and OFDMA have been selected by 3GPP as the physical layer and multiple access schemes for DL LTE. Because of the high degree of flexibility in the allocation of radio resources to UEs, the optimization of this process can become very complex. In order to achieve a high frequency reuse, the problem of RRM must be addressed jointly with a certain inter-cell interference coordination (ICIC) and from a multi-cell perspective. Considering the DL, the key RRM function is the scheduling of DL transmissions to the different users performed at the eNode-B (eNB) at both Time Domain and Frequency Domain [2], where time is divided into $1 \mathrm{~ms}$ Transmission Time Intervals (TTI) and frequency into $180 \mathrm{kHz}$ Physical Resource Blocks (PRB). Ideally, ICIC should be performed as decentralized as possible so that no new signalling is introduced, however this usually leads to suboptimal ICIC. In particular, the most popular approaches that are usually found in the literature are:

a) Those assuming a fixed and static reuse pattern depending on the UE measured Signal to Interference and Noise Ratio (SINR) or Channel Quality Indicator (CQI) or other measured or estimated parameter [3].

b) Those without prefixed bandwith partitions but still using some sort of centralized entity co-located at one of the eNBs, the Central Control Unit CCU [4]. The role of the $\mathrm{CCU}$ is to gather measurements from several neighbouring eNBs in order to coordinate their scheduling processes on a coarse time scale.

This work has been supported by the Spanish National Science Council under grant TEC2008-06817-C02-02 and by the European Regional Development Fund (ERDF).
In addition to ICIC several other aspects, like guaranteeing the Quality of Service (QoS) of connections, signalling overhead minimisation, fairness among users, adaptation to traffic patterns and implementation issues, need to be addressed in the RRM design at eNB level. The resulting problem is very complex and it is often intractable in an analytic way. So eventually the different proposals are assessed by means of system level simulations under as realistic as possible scenarios.

The aim of this paper is to evaluate the performance of a multi-cell radio resource allocation methodology aiming at improving ICIC under the LTE framework and considering a semi-distributed approach. The methodology that has been followed is the use of MonteCarlo simulations in which the system level simulator is supported by a detailed E-UTRA link level simulator to perform Link Adaptation (LA) through Adaptive Modulation and Coding (AMC) including Hybrid ARQ (HARQ), a Multipath Fading Channel and MIMO.

The paper is organized as follows: section II provides a detailed explanation of the proposed RRM from both super frame and TTI perspective. System model and problem formulation are also presented in section II. Section III discusses the simulation results and finally section IV addresses future work and conclusions.

\section{DESCRIPTION OF THE MULTI-CELL RRM PROPOSAL}

In this section we describe the multi-cell scheduling framework and the assumptions that have been made. The proposed semi-distributed scheme coordinates inter-cell interference with a coarse time resolution (a super-frame (SF)) at the CCU, while fairness and throughput maximization are controlled within a smaller time scale by the eNB decentralized scheduling algorithm (TTI RRM), see Figure 1.

A PRB can only be assigned to one user equipment (UE) within a cell, but neighbouring cells may reuse the same PRB depending on the UE interference level. Moreover, depending on the global bandwidth the minimum number of simultaneous PRBs that can be assigned to a UE varies between 1 and 4 (in that so called Resource Block Group, RBG). 


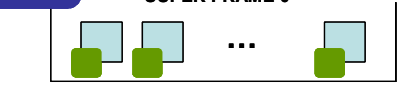

SUPER FRAME $K$
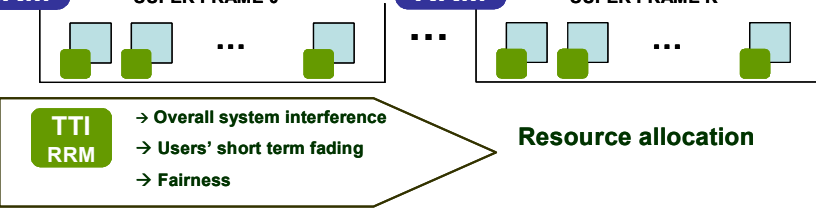

Figure 1. Temporal structure of the RRM scheme

\section{A. Central Control Unit: Super Frame RRM}

CCU problem consists in assigning a number of RBGs (and the transmitted power on that RBG if variation is allowed) to the eNBs so that the global system throughput is maximized.

$$
\max \left(\sum_{n=1}^{N_{R B G}} T H R_{n}\right)=\sum_{n=1}^{N_{R B G}} \sum_{i=1}^{M} T H R_{i n}
$$

Being $N_{R B G}$ the number of RBGs in the considered bandwidth, $T H R_{n}$ the system throughput associated to RBG $n$, $M$ the total number of UEs in the system distributed along the $B$ eNBs considered in the analysis, and $T H R_{\text {in }}$ the throughput of UE $i$ on RBG $n$. CCU makes decisions at Super-Frame level oriented to coordinate a set of neighbouring eNBs to reduce and bound the inter-cell interference between cells. The CCU has available, at the beginning of each super-frame, the CQI's per RBG of all the UEs served by the set of coordinated eNBs, as well as the estimation of the path loss of the dominant interferers per UE. Up to 6 interferers, estimated through pilots are considered in the simulator. In each TTI, each UE computes the Exponential Effective Signal to Interference and Noise Ratio (EESINR) to obtain its CQIs for all the RBGs. To do so, in the case of $20 \mathrm{MHz}$ bandwidth it is necessary to obtain, ideally for each sub-carrier $k$, the instantaneous $\operatorname{SINR}_{k}$ at the system level simulator [10] which in turn depends on the average SINR. The $\overline{\operatorname{SINR}_{l n}}$ measured by user $i$ on RBG $n$, associated to the serving $\mathrm{eNB} \mathrm{j}$ is calculated as:

$$
\overline{S I N R_{i n}}=\frac{P_{j n} / L_{i \hat{i}}}{\sigma_{R B G}^{2}+\sum_{\substack{b=1 \\ b \neq j}}^{B}\left(\sum_{m=1}^{M_{b}} y_{m n} \cdot P_{b n} / L_{i b}\right)}
$$

Being $\sigma_{R B G}^{2}$ the UE received thermal noise at RBG $n, M_{b}$ the number of UE served by eNB $b$, so that $M=\sum M_{b}, y_{m n}$ is the UE-RBG assignment variable, which is 1 if user $m$ obtains RBG $n$, and 0 otherwise. The power transmitted by eNB $j$ on RBG $n$ is given by $P_{j n}=\phi_{j n} \cdot P_{T, t o t}^{\max } / N_{R B G}=\phi_{j n} \cdot P_{T, R B G}^{\max }$, being $P_{T, t o t}^{\max }$ the total eNB available output power and $P_{T, R B G}^{\max }$ the maximum transmitted power per each RBG. Moreover, $L_{i j}$ is the long term channel gain between eNB $j$ and user $i$, while $L_{i \hat{\imath}}$ is the long term channel gain between user $i$ and its serving eNB $\hat{\imath}$. Finally, $\phi_{j n} \in[0,1]$ represents the fraction of the maximum power per RBG that eNB $j$ has decided to assign to RBG $n$. It is initially assumed that all eNBs transmit at full power, equally distributed among all RBGs, so $\phi_{j n}=1$.
Following [10], the EESIR is obtained by:

$$
E E S I R=-\beta \ln \left(\frac{1}{N_{u}} \sum_{k=1}^{N_{u}} e^{-\frac{S I N R_{k}}{\beta}}\right)
$$

Being $N_{u}$ the number of useful subcarriers in a RBG (for 20 $\mathrm{MHz}$ bandwidth a RBG is composed of 4 PRBs and each PRB has 12 subcarriers, so $N_{u}$ is equal to 48 subcarriers, neglecting pilots). Since there are 15 different values of $\beta$, each one corresponding to a different combination of modulation and coding (look up tables from the link layer simulator), there will be 15 different values of EESSIR. There is also a mapping between EESIR and BLER. The UE should start calculating the EESSIR for the combination of maximum throughput (maximum modulation order and higher coding rate). If for this combination BLER $\leq 0.1$ it is not necessary to obtain the values for the other fourteen. If the BLER $>0.1$ the procedure is repeated with the next modulation and code combination, and so on. With this procedure what is finally obtained is that, for each $\mathrm{RBG}$, the UE reports to the eNodeB a CQI which is an index between 0 and 15 ( 0 means out of range) that gives the information about the highest modulation and coding scheme that can be used in each RBG. This is the information used at the $\mathrm{CCU}$ to assign resources to the different eNBs.

This mapping function has been obtained from link level simulations following the actual settings given in LTE specs. The H-ARQ mechanism and fast fading channel are included in link level simulations as well as MIMO.

The CCU decides the set of RBGs assigned to each eNB for the next super-frame and recommends the number of RBGs to be assigned to the specific UEs by estimating and bounding the inter-cell interference through the Throughput Marginal Utility (TMU) of the users, [4]. The algorithm is clearly oriented to maximize the system throughput. In particular, once the CCU knows the $\mathrm{CQI}_{\text {in }}$ of user $i$ over RBG $n$, it can establish a direct mapping with the maximum throughput $T H R_{i}$ that can be assigned to $i$. The system tries to equalize the number of RBGs (and therefore the global throughput), thus cell-edge users can receive more RBGs to compensate for their higher path loss and interference levels. Hence $n_{i}$ values represent an upper bound on the number of RBGs that user $i$ can receive.

$$
\mathrm{n}_{1} \mathrm{THR}_{1}=\mathrm{n}_{2} \mathrm{THR}_{2}=\cdots=\mathrm{n}_{\mathrm{M}_{\mathrm{b}}} \mathrm{THR}_{\mathrm{M}_{\mathrm{b}}}
$$

$$
\text { Being } \sum_{i=1}^{M_{b}} n_{i}=N_{R B G}
$$

It's important to notice that the algorithm finds different frequency reuse patterns depending on the average SINR of each UE or more specifically depending on the CQI values reported by them. Those users with the highest CQI will be considered as internal and for them a reuse 1 will be applied. Therefore when considering the set of RBGs assigned to each eNB, only the rest of UEs will be included in the algorithm, these are named intermediate and cell-edge UEs. This classification in terms of CQI is quite similar to the mapping obtained through assigning thresholds to the average SINR.

Next step is a heuristic RBG allocation which deals with inter-cell interference and tries to maximize the system 
throughput by means of three nested loops. Basically, the algorithm assigns PRBs one by one to the different eNBs considering the degradation on the other eNBs to which that PRB has been already assigned.

- The first loop (external) evaluates RBGs one by one.

- Next is associated to eNBs (1...B). The order in which they are checked is inversely proportional to the number of PRBs already assigned to them. This avoids benefitting any particular cell.

- Finally, the third one assesses the users inside the considered eNB $\left(1 \ldots \mathrm{M}_{\mathrm{b}}\right)$.

Thus, the evaluated RBG is assigned to a specific UE inside a specific eNB if:

- The number of RBGs already assigned to the user is lower than $n_{i}$ (the maximum number in terms of fairness).

- If this allocation improves the global throughput. In order to obtain this metric, it is necessary an estimation of the interference increase and for this reason path losses between UEs and their stronger interferers is required.

\section{B. TTI RRM}

The final resource allocation is done at each eNB, which is based on recommendations given by the $\mathrm{CCU}$ algorithm. The eNB decides the pairing between RBGs and UEs considering their instantaneous channel conditions (and thus the instantaneous throughput) and the average throughput of each user, that means a classical Proportional Fair scheduling algorithm is applied in each RBG $n$ :

$$
i=\operatorname{argmax} \frac{T H R_{i, n}(t)}{\sum_{k=1}^{t-1} T H R_{i, n}(k)} \quad 1 \leq i \leq M_{b}
$$

Also constrains from the CCU RRM algorithm on the maximum number of PRBs to be granted for each specific UE are taken into account.

The eNB is also responsible for applying the scheduling rules to the different type of users: internal, intermediate and cell-edge, which are sequentially attended (one TTI per type of user) following a Round Robin scheme. As previously stated, internal UEs are able to use all PRBs (reuse 1), however intermediate and cell-edge ones can only use those indicated by the CCU. In general, available PRBs for external users in neighbouring cells will tend to be orthogonal among them.

If traffic is not balanced between the three different types of UEs the fixed temporal scheduling can be very inefficient. To save this, if the eNB detects that more than a half of the available RBGs are not going to be used by the internal UEs, it can assign these extra resources to some intermediate ones. Since these users are served out of their temporal slot, they will perceive a higher interference and their throughput will not be optimal, however this is preferable to wasting available resources. A refinement to this would be to soften the strategy by considering adjustable incremental thresholds (with some limits) to adapt to different UEs distribution and traffic requirements.

\section{SimUlation RESUlTS AND DisCUSSION}

In order to feed the system level simulator with the link level performances, a new ad-hoc link level simulator has been programmed in $\mathrm{C}++$ language. The E-UTRA DL link level simulator features an OFDM physical layer in accordance with [9], and has been completely described in [10].

Table I lists the parameters used for the simulations. In order to achieve a high time resolution, the simulation uses the maximum bandwidth, but only one PRB is demodulated by the UE. The simulated code block sizes are the smaller ones specified for E-UTRA DL. The obtained link level throughput can thus be considered the E-UTRA DL baseline performance, since higher code block sizes will provide higher throughput figures.

The considered channel model is Extended Pedestrian A (EPA), as specified in [8], with a $3 \mathrm{~km} / \mathrm{h}$ pedestrian speed.

TABLE I. LINK AND SYSTEM LEVEL SIMULATOR PARAMETERS

\begin{tabular}{|c|c|}
\hline Parameter & Value \\
\hline Carrier frequency & $2 \mathrm{GHz}$ \\
\hline Transmission Bandwidth & $20 \mathrm{MHz}$ \\
\hline Sub-carrier spacing & $15 \mathrm{kHz}$ \\
\hline OFDM PHY parameters & $\begin{array}{c}\mathrm{CP} \text { of } 4.69 \mu \mathrm{s} \\
7 \text { modulation symbols/sub- } \\
\text { frame }(2 \text { for control })\end{array}$ \\
\hline FFT size & 2048 \\
\hline Number of useful sub-carriers & 1200 \\
\hline OFDM symbol duration & $71.43 \mu \mathrm{s}$ \\
\hline Number of sub-carriers per PRB & 12 \\
\hline Number of PRBs/RBGs & $100 / 25$ \\
\hline Sub-frame duration & $0.5 \mathrm{~ms}$ \\
\hline $\begin{array}{c}\text { TTI length } \\
\end{array}$ & $1 \mathrm{~ms}$ \\
\hline Number of OFDM symbols per TTI & 14 (4 for control) \\
\hline Frame duration & $10 \mathrm{~ms}$ \\
\hline Superframe duration & $200 \mathrm{~ms}$ \\
\hline Transmission mode & Localized \\
\hline Power Delay Profile & $\begin{array}{c}\text { EPA channel model } \\
\text { Pedestrian speed } 3 \mathrm{~km} / \mathrm{h}\end{array}$ \\
\hline Channel Coding & Turbo code basic rate $1 / 3$ \\
\hline Code block sizes & $40-120$ bits \\
\hline Rate Matching and H-ARQ & $\begin{array}{l}\text { According to [9] (release } 8 \text { ). } \\
\text { Max } 4 \text { IR transmissions. }\end{array}$ \\
\hline AMC formats & $\begin{array}{c}\text { QPSK: } 1 / 3,1 / 2,2 / 3,4 / 5 \\
\text { 16QAM: } 1 / 2,2 / 3,4 / 5 \\
\text { 64QAM: } 2 / 3,4 / 5\end{array}$ \\
\hline Channel estimation & Ideal \\
\hline Antenna scheme & SISO/MIMO \\
\hline Cell radius & $500 \mathrm{~m}$ \\
\hline Path loss expression & $31.5+35 \log (\mathrm{d}[\mathrm{m}])[\mathrm{dB}]$ \\
\hline $\begin{array}{c}\text { Shadowing fading standard } \\
\text { deviation }\end{array}$ & $7 \mathrm{~dB}$ \\
\hline $\begin{array}{l}\text { Number of active UEs per cell } \\
\text { (infinite buffer per user) }\end{array}$ & 15 \\
\hline Number of cells & $\begin{array}{c}57 \text { sectorial (statisctics } \\
\text { collected from the internal } \\
21 \text { ) }\end{array}$ \\
\hline
\end{tabular}

Propagation losses account for path loss, lognormal shadowing and building penetration losses (if indoor users are considered). 
Figure 2 shows the E-UTRA DL throughput for the different AMC formats and H-ARQ in EPA multipath channel at a pedestrian speed of $3 \mathrm{~km} / \mathrm{h}$ and for a $2 \times 2$ MIMO MMSE system with low antenna correlation.

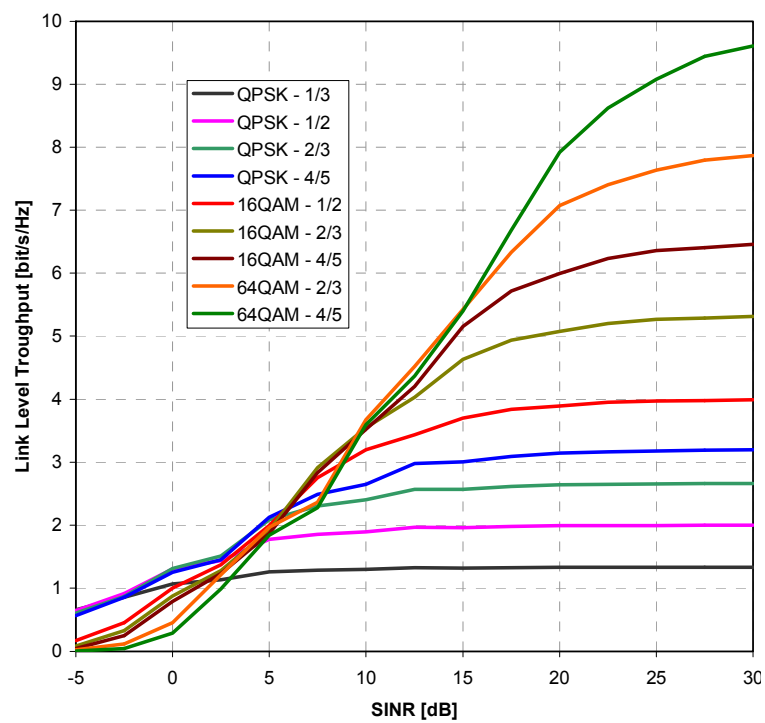

Figure 2. UTRA DL AMC link level throughput with H-ARQ in multipath EPA channel $3 \mathrm{~km} / \mathrm{h}$ with $2 \times 2$ MIMO MMSE

Other figures similar to Figure 2 and used in this paper are given and explained in detail in [10]. Figure 3 is a histogram of the SINR distribution perceived by the UEs in the 21 central cells of the scenario. The thresholds that have been set up in order to classify the UEs in three different classes for scheduling purposes are:

\section{External UEs: SINR $\leq 1 \mathrm{~dB}$ \\ Intermediate UEs: $1 \mathrm{~dB}<\mathrm{SINR} \leq 7.5 \mathrm{~dB}$ \\ Internal UEs: $7.5 \mathrm{~dB}<\mathrm{SINR}$}

As uniform users distribution is considered it can be observed that approximately $33 \%$ of users have a SINR lower than $1 \mathrm{~dB}$ (external users), 33\% experience a SINR between 1 and $7.5 \mathrm{~dB}$ (intermediate users), having the rest a SINR higher than $7.5 \mathrm{~dB}$ (internal users) but in this case showing a great dispersion in the SINR value (lower slope) that is expected to become a higher difference in accepted throughput values for this kind of users when compared with the rest.

In Figure 4 the histograms of the average cell throughput and the corresponding cdf is given for SISO and MIMO systems with different antennas correlation. The histogram is composed of three clearly differentiated parts (sub-histograms), consistent with the fact that eNBs serve the different classes of users sequentially (one class per TTI) and in a coordinated way. Besides, the reason for the clear differentiation among the three sub-histograms is two-fold. First, cell-edge and intermediate users cannot use the whole bandwidth, with the correspondent throughput degradation. The more external the users are, the lower the number of available PRBs. Second, when users experience poorer channel conditions, the eNB selects transport formats in which the effective payload is lower. For example, since cell-edge users report lower CQIs
SINR their connection will use a lower order modulation (QPSK) and coding rate, while the internal ones are likely to use 64QAM and coding rates close to one.

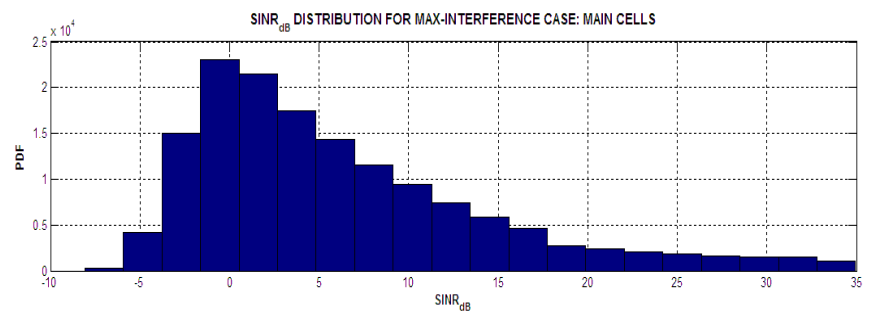

CDF: SINR ${ }_{\mathrm{dB}}$ FOR MAX-INTERFERENCE CASE: MAIN CELLS

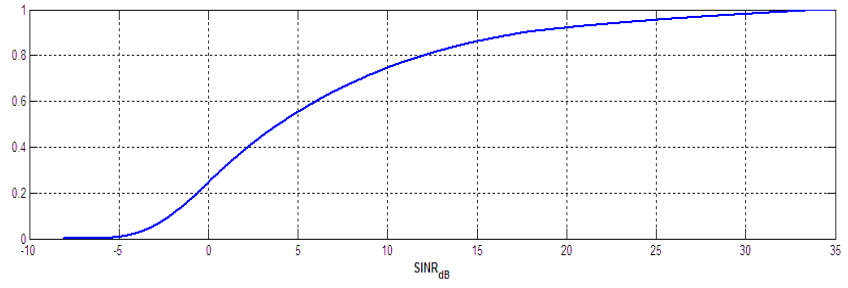

Figure 3. SINR perceived by the UEs in the 21 central cells of the scenario.

Next to each sub-histogram it can be appreciated three vertical lines showing the throughput upper bound for each user class. This is a useful indicator to assess the goodness of the global system strategy, that is to say the particular combination of ICIC and distributed scheduling. Each line is computed considering the maximum spectral efficiency $\mathcal{E}$ (in $\mathrm{bps} / \mathrm{Hz}$ ) of a particular user class. So, the three values are the result of multiplying $\varepsilon$ by the global bandwidth $(20 \mathrm{MHz})$ and multiplying also by 10/14 (considering 10 useful symbols from the 14 symbols of each TTI) and dividing by 3 (temporal scheduling) and are listed in Table II

TABLE II: ASYMPTOTIC MAXIMAL THROUGHPUT

\begin{tabular}{|c|c|c|c|}
\hline System & Type of UEs & Asymptotic THR & CellTHR \\
\hline \multirow{3}{*}{ SISO } & Internal & 18.7 & \multirow{3}{*}{25.53} \\
\hline & Intermediate & 5.57 & \\
\hline & External & 2.26 & \\
\hline \multirow{3}{*}{$\begin{array}{c}\text { MIMO } \\
\text { (high correlation) }\end{array}$} & Internal & 25 & \multirow{3}{*}{33.57} \\
\hline & Intermediate & 5.78 & \\
\hline & External & 3.64 & \\
\hline \multirow{3}{*}{$\begin{array}{l}\text { MIMO } \\
\text { (medium } \\
\text { correlation) }\end{array}$} & Internal & 33.86 & \multirow{3}{*}{44.35} \\
\hline & Intermediate & 6.21 & \\
\hline & External & 4.28 & \\
\hline \multirow{3}{*}{$\begin{array}{c}\text { MIMO } \\
\text { (low correlation) }\end{array}$} & Internal & 40.48 & \multirow{3}{*}{54.83} \\
\hline & Intermediate & 9 & \\
\hline & External & 5.35 & \\
\hline
\end{tabular}

Another aspect that can be clearly appreciated is that there is a higher dispersion in the throughput associated to high SINR users. This is logical, considering that SINR dispersion is higher in internal users, followed by the intermediate ones. As expected, the CDF function clearly shows the three steps associated to the three sub-histograms, with jumps around $33 \%, 66 \%$ and $99 \%$, consistent with the previously defined thresholds to classify users in each SF. As the dispersion increases when SINR is higher, the slope of the step is progressively reduced. 

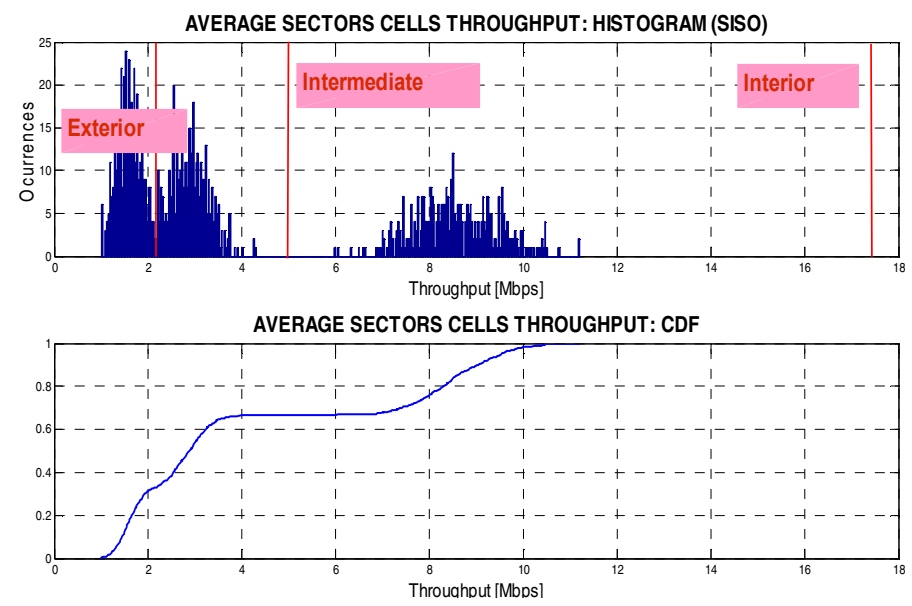

(a)

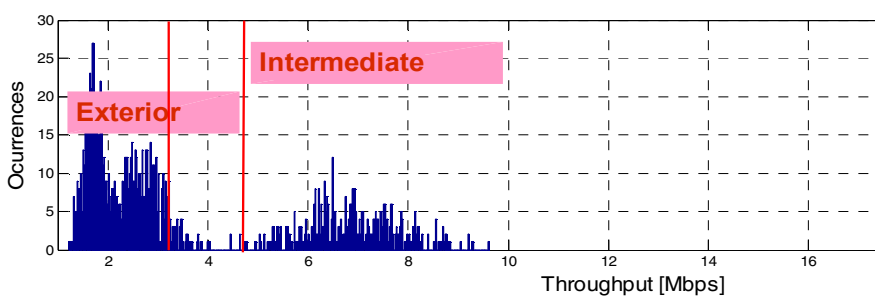

AVERAGE SECTORS CELLS THROUGHPUT:

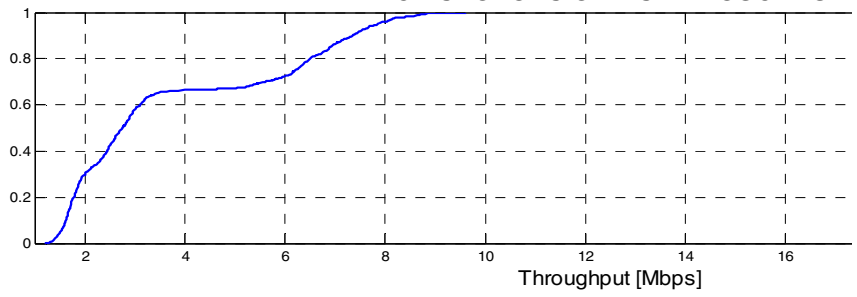

(b)

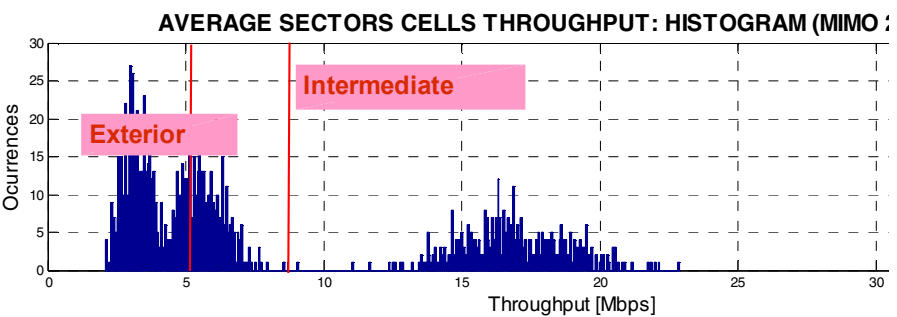

AVERAGE SECTORS CELLS THROUGHPUT: CDF

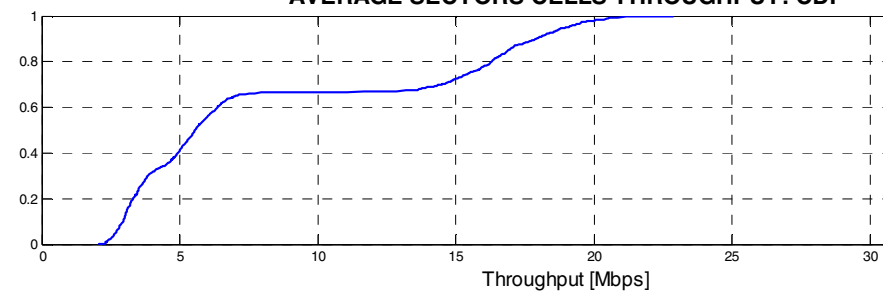

Figure 4: Histogram and CDF of the DL average cell throughput (a) SISO, (b) $2 \times 2$ MIMO MMSE/SFD with high correlated antennas, (c) $2 \times 2$ MIMO MMSE/SFD with low correlated antennas

\section{CONCLUSIONS AND FUTURE WORK}

3GPP specifications do not include scheduling schemes, giving the vendors and operators the opportunity to define their own proposals. So it is a field where intensive research activity has been done in last year's. The scheme proposed and analyzed here is a simple approach to both time and frequency scheduling, which allows a dynamic adaptation of the SINR thresholds or CQI values defining the UEs classes, oriented to a better sharing of the time resource and a cell throughput increase. AMC scheduling is directly done by trying to assign each UE the RBG that maximizes its efficiency (the one associated to the highest combination of modulation and coding parameters). Finally, frequency scheduling is initially done by the Central Control Unit assuring that neighbor cells don't use simultaneously the same RBG for intermediate and cell edge UEs, while the detailed assignment is done on a eNB basis, because this internal and fast scheduling can be aware of channel variations facilitating a fair assignment of resources. $\mathrm{CCU}$ algorithm can be performed without requiring significant extra signaling.

To continue testing some of the facilities of the algorithm, specially the dynamic adapting of the thresholds or UEs regions as well as the variation of the transmitted power by region, it is necessary to implement real traffic modeling instead of infinite buffer UEs. Another interesting option that will be tested is the possibility to dynamically extend/modify UEs regions if the UEs belonging to a given region do not use all the resources assigned to them (especially intermediate UEs can move use internal RBG, and external UEs could use intermediate RBGs). This again has to be tested under real traffic conditions to obtain reliable results. With this we could have a single algorithm at the CCU/eNBs which depending on traffic and other measures, could dynamically choose to adapt some parameters to maximize throughput, or quality.

\section{REFERENCES}

[1] 3GPP TR 25.814, "Physical Layer Aspects for E-UTRA" (Release 7), v7.1.0

[2] Niko Kolehmainen et al, "Channel Quality Indication Reporting Schemes for UTRAN Long Term Evolution Downlink", IEEE Vehicular Technology Conference, VTC spring 2008

[3] Haipeng LEI et al, "System Level Evaluation of 3G Long Term Evolution" PIMRC'07

[4] G. Li, H. Liu, "Downlink Radio Resource Allocation for Multi-Cell OFDMA System" IEEE Trans. Wireless Commun., vol. 5, pp. 34513459, Dec. 2006

[5] S-E. Elayoubi et al, "Performance Evaluation of Frequency Planning Schemes in OFDMA-based Networks", IEEE Transactions on Wireless Communications, vol. 7, no. 5, may 2008

[6] C. Koutsimanis, "Inter-Cell Interference Coordination Techniques for Multi-Cell OFDMA Networks Supporting Narrow Band and Elastic Services". Master of Science Thesis. Royal Institute of Technology (KTH), May. 2007

[7] Tse Viswanath, "Fundamentals of Wireless Communications" Cambridge University Press, 2005.

[8] 3GPP TR 36.804, "E-UTRA Base Station (BS) radio transmission and reception" (Release 8), v1.2.0

[9] 3GPP TS 36.212, "E-UTRA Multiplexing and Channel Coding" (Release 8), v8.3.0

[10] J.Olmos et al,"Link_Level_Simulator_for_LTE_Downlink" COST2100 $\operatorname{TD}(09) 779$, Braunschweig, February 2009

[11] 3GPP TR 25.892 "Feasibility Study for Orthogonal Frequency Division Multiplexing (OFDM) for UTRAN enhancement 